\title{
ANÁLISE DA UTILIZAÇÃO DO BSC EM EMPRESAS DE SEGMENTOS DISTINTOS
}

\section{ANALYSIS OF UTILIZATION OF THE BSC IN COMPANIES OF DISTINCT SEGMENTS}

\author{
João Francisco Saraiva Puglisi, Mateus Seribeli Neto, Porfirio Henrique de Sá \\ Filho, Rafael Medeiros Hespanhol
}

Universidade do Oeste Paulista - UNOESTE, Faculdade de Engenharia, Presidente

Prudente, SP.

E-mail: mateus.seribeli@gmail.com

RESUMO - As empresas contemporâneas estão confrontando um cenário altamente competitivo e dinâmico, a estratégia empresarial tem papel fundamental em preparar as organizações para que as mesmas cresçam em seu segmento, sendo direcionadas a seus objetivos globais, e assim sobreviverem neste ambiente cheio de incertezas. Existem diversas ferramentas que auxiliam a implantação e gestão das estratégias empresariais, como o Balanced Scorecard (BSC), que aborda acompanhamento dos indicadores relacionados ao desempenho da organização no alcance de suas estratégias, norteando as ações dos colaboradores mediante a estratégia adotada. O objetivo do presente trabalho constitui-se em reunir estudos de caso de empresas de segmentos diferentes que tenham aplicado a ferramenta BSC para então analisar as diferentes formas de abordagem desta ferramenta para cada estudo que foi apresentado e verificar a peculiaridade de cada caso. Palavras-chave: Estratégia empresarial; Ferramenta de Gestão; BSC; Estratégia Corporativa; Visão.

ABSTRACT - The contemporary companies are confronted with a highly competitive and dynamic scenario, the corporate strategy has a fundamental role in preparing organizations to grow in their segment, being directed to their global objectives, and thus survive in this environment full of uncertainties. There are several tools that help the implementation and management of business strategies, such as the Balanced Scorecard (BSC), which addresses the follow-up of indicators related to the organization's performance in reach of his strategies, guiding employees actions through the strategy adopted. The purpose of this study is to gather case studies of companies from different segments that have applied the tool BSC to analyze the different ways of approach of this tool for each study that was presented and verify the peculiarity of each case.

Keywords: Business Strategy; Management Tool; BSC; Corporate Strategy; Vision. 


\section{INTRODUÇÃO}

Para Bhalla et al. (2009) o conceito de estratégia apresenta-se ainda muito vago, passível de várias interpretações. Conforme Carvalho e Laurindo (2010) Von Clausewitz, desempenhou um papel que fundamental no desenvolvimento do conceito de estratégia militar, sendo que para este, a estratégia apresentava um caráter descritivo que parte da ideia de que não há um caminho prédeterminado para o sucesso estratégico, porém pode-se aprender por meio de experiencias anteriores, sendo que esta é a visão da estratégia mais relevante dentre as existentes nos tempos atuais. $\mathrm{O}$ autor ainda complementa que elaborar uma estratégia ou assumir uma posição estratégica, significa executar atividades diferentes das de seus concorrentes, ou seja, significa atuar de forma única em vista de que as situações são únicas e dinâmicas e que por meio desta teoria é que as empresas conseguirão perpetuarem durante um longo período.

Considerando que atualmente as organizações se encontram num mercado altamente competitivo e dinâmico, Mainardes, Ferreira e Raposo (2011) relatam que a estratégia representa um importante papel na gestão empresarial tendo como principal finalidade preparar a organização para sobreviver e se desenvolver em meio a este ambiente, usando para tal, das qualificações, recursos e competências da empresa. Esta dinamicidade de acordo com Lima (2009) tem exigido das empresas o constante uso da estratégia, impondo assim diversos desafios as empresas como por exemplo, fazer trade-offs entre escolher entre reduzir de maneira defensiva, manter ou aumentar o seu escopo corporativo, escolha que envolve um grande esforço por parte da organização como um todo. Após a organização realizar as escolhas e elaborar a estratégia se faz necessário verificar se os níveis da empresa estão atuando de modo que contribua para o alcance da estratégia definida, para tal, Carvalho e Laurindo (2010) relata que o Balanced Scorecard (BSC) consiste em uma abordagem que indica o desempenho da organização no atendimento de suas estratégias.

O objetivo da presente investigação consiste em buscar reunir estudos de caso que tenham aplicado a ferramenta BSC demonstrando assim as diferentes formas de abordagem desta ferramenta.

\section{CARACTERIZAÇÃO DO BSC}

O BSC foi originado nos Estados Unidos na década de 1990, e os relevantes precursores foram Robert Kaplan e David Norton, que com a colaboração de outros pesquisadores averiguaram que os indicadores, principalmente financeiros, cujo as organizações se utilizavam apresentavam-se insuficientes para ampara-las a criarem valores econômicos futuros. Concluem que a partir da visão e da estratégia e definindo indicadores de performance que expressassem essa estratégia, de modo estruturado em quatros interpretações (Financeira, dos Clientes, dos Processos Internos e de Aprendizado e Crescimento), as organizações assim obtiveram um sistema amplo de avaliação (FARIA; DA COSTA, 2007).

Em 1997, o BSC foi apontado, pela obra impressa Harvard Business Review, sendo uma das ideias gerenciais mais relevantes dos últimos 75 anos (SIBBET, 1997). Importante ressaltar que de acordo com Oliveira (2008) a General Electric (GE) implementou nos anos 50 algo como uma ideia do BSC, pois esta ferramenta foi criada por David Norton e Robert Kaplan no início dos anos 90.

No Brasil, o conceito foi formalizado por meio da tradução para o país no ano 2000, através dos trabalhos da consultoria Symnetics Ltda, quando instituiu uma parceria juntamente com a BSC Collaborative (BSCOL), empresa fundada por Robert Kaplan e David Norton. (OLIVEIRA, 2008). Desde os estudos iniciais, em 1992, o BSC vem obtendo progressiva popularidade como ferramenta de gestão que norteia ações dos funcionários e metas à estratégia corporativa (DAVIS; ALBRIGHT, 2004)

Kaplan e Norton (1997) conceberam possivelmente uma das ferramentas mais empregadas para a avaliação da performance nas empresas, sendo o BSC. É uma ferramenta de fácil entendimento e ainda proporciona uma melhor exposição das estratégias, pois reuni as informações em planilhas e mapas apresentando a influência das ações umas nas outras, ou seja, a correlação de causa e efeito. O BSC é um conceito de gestão que estabelece o estreitamento do gap ${ }^{1}$ existente em meio a estratégia e a parte operacional do negócio, norteando a organização aos seus objetivos estratégicos.

Contudo, o conceito de BSC não é inerte. Evidencias empíricas indicam que os utilizadores do BSC por diversas vezes iniciam com um 
scorecard superficial e rustico, que é aperfeiçoado em suas funcionalidades e em seu escopo de execução com o passar do período. Isso acarreta no fato que o BSC está distante de ser um conceito definido (SPECKBACHER; BISCHOF; PFEIFFER, 2003).

Kaplan e Norton (2000) afirmam que as quatro perspectivas devem responder questões básicas: como somos vistos por nossos clientes? (Dimensão do cliente); em que devemos ser os melhores? (Dimensão interna); como atingir a visão, mantendo o potencial de crescer e inovar? (Dimensão do aprendizado e crescimento); como somos vistos por nossos acionistas? (Dimensão financeira). Na perspectiva clientes, se define quais segmentos de mercado irá se competir para identificar as medidas de desempenho que pertencem ao setor, possibilitando um benchmark que auxilia a geração de metas, percebendo como a organização é vista pelos clientes. Na perspectiva dos processos internos sugere-se no que a empresa deve melhorar, tendo medidas que servem para impulsionar o aperfeiçoamento nos processos que são críticos para realizar a estratégia estabelecida, focando nos processos determinantes no sucesso da estratégia da empresa.

São estabelecidos na perspectiva do aprendizado e crescimento uma infraestrutura que suporte os objetivos que serão elaborados pelos processos internos, para atingir a visão e manter o potencial de crescimento e inovação. Os elementos desta perspectiva são: capacidade dos funcionários; capacidades dos sistemas de informação e o alinhamento dos procedimentos e rotinas organizacionais. Por fim existe a perspectiva financeira, que traduz monetariamente as metas de longo prazo. Com foco em gerar retornos superiores ao capital investido, mostrando a importância do BSC para os acionistas. E a partir deste enfoque, são dados os objetivos e medidas para todas outras três perspectivas do balanced scorecard. Portanto toda medida adotada para um scorecard deve estar dentro de uma cadeia de relações de causa e efeito que termina com objetivos financeiros e, representa um tema estratégico para a empresa (BIASSOTO, 2006).

Segundo Back, Dutra e Casagrande (2012) a estrutura do BSC é composta por: um mapa estratégico, que descreve a estratégia através dos objetivos relacionados entre todas perspectivas; um objetivo estratégico, que define aquilo que a organização quer alcançar; o indicador, que tem a função de medir e acompanhar o sucesso da estratégia organizacional; metas, que se referem ao nível de desempenho ou à taxa de melhorias que têm de ser realizadas para que os objetivos sejam alcançados; e pelo plano de ação, que pauta as ações práticas necessárias para atingir os objetivos estratégicos da empresa.

Os objetivos estratégicos traduzem a visão da empresa em uma relação de causa e efeito, que é composto por um mapa estratégico. $E$ esse mapa contribui para que todos os indivíduos na organização entendam a estratégia da empresa e transformem ativos intangíveis em resultados tangíveis (KAPLAN \& NORTON, 2000). Assim Kaplan e Norton (1997) destacam que um bom balanced scorecard deve ser uma combinação adequada de resultados com impulsionadores de desempenho ajustados à estratégia. Onde os resultados são representados pelos indicadores de ocorrência, que mostram o desempenho das ações passadas, e os impulsionadores de desempenho são representados pelos indicadores de tendência, que servem para indicar quais serão os prováveis resultados futuros.

\section{APLICAÇÕES DO BSC}

A presente pesquisa para atingir seu objetivo, realizou uma pesquisa bibliográfica na busca por estudos de casos que abordaram a ferramenta BSC nas organizações que serão apresentados e discutidos adiante.

Borges, Almeida e Silveira (2011) em seu trabalho intitulado "O Balanced Scorecard aplicado no mestrado acadêmico de Administração da Universidade Estadual do Ceará - UECE." tem como objetivo demonstrar a aplicação da metodologia pelos conceitos que fundamentam o BSC como ferramenta estratégica útil para a condução do curso de Mestrado Acadêmico em Administração da Universidade Estadual do Ceará. É relevante abordar que nesta pesquisa houve uma adaptação da ferramenta BSC, sendo que ao invés de abordar a perspectiva financeira, esta foi substituída por uma perspectiva que de acordo com o autor se adequa melhor ao contexto educacional, sendo denominada de perspectiva de desempenho acadêmico. Essa modificação se justifica pelas características dos programas de cursos de Pós-graduação Stricto Sensu das Universidades públicas que são avaliados com uma determinada periodicidade, podendo obter 
notas de um a quatro, as quais definem a qualidade do curso.

Com relação ao uso da ferramenta $B S C$ o artigo trouxe na perspectiva de Aprendizado como objetivos: Ministrar disciplinas e conteúdos com qualidade; motivar os mestrandos a produção científica; estimular a participação de Professores/Alunos/Funcionários em cursos e treinamentos; promover a participação dos alunos/Professores em congressos/seminários; realizar Benchmark em outros cursos de Mestrado da área. E como indicadores para apontar o alcance ou não alcance dos objetivos, optaram-se respectivamente: Avaliação dos professores pelos alunos; número de artigos publicados por ano de alunos do mestrado; número de participações em cursos e treinamentos; número de participações em eventos científicos; número de professores convidados ou visitantes.

Por conseguinte, na perspectiva de Processos Internos foi estabelecido como objetivos: atender eficientemente a demanda dos professores e alunos no trabalho de produção científica; disponibilizar material didático e de pesquisa aos professores/alunos; apresentar estrutura curricular adequada à formação do pesquisador em Administração. Como indicadores, respectivamente optou-se por: Número de reclamações à coordenação por semestre; número de livros e periódicos por aluno e por professor; número de alterações curriculares por ano.

$\mathrm{Na}$ perspectiva dos clientes foram considerados como objetivos: promover a maior satisfação possível dos alunos com os recursos disponíveis; atender as demandas da sociedade cearense por conhecimento na área do curso; promover a maior satisfação possível dos professores com os recursos disponíveis. Como indicadores a estes objetivos: Índice de satisfação dos alunos; número de projetos e consultas realizadas ao curso pela sociedade cearense; índice de satisfação dos professores.

Por fim, com relação a perspectiva de desempenho acadêmico foram elencados como objetivos: elevar o número de publicação discente; elevar o número de publicações dos docentes; obter um número satisfatório de professores do NRD6 para o atendimento das necessidades dos mestrandos; oferecer ao ambiente acadêmico um número satisfatório de alunos titulados pelo curso. E com relação aos indicadores, respectivamente serão: número de publicações discentes por número de alunos matriculados; número de publicações docente por número de Prof. no NRD6(1); número de docentes NRD6 por número de alunos matriculados; média titulados por ano pelo número de Professores no NRD6.

Borges, Almeida e Silveira (2011) relatam que os objetivos que não possuem metas não atingidas podem, pela sequência de causa e efeito do BSC, mostrar que caminho o gestor deve seguir para resolver seus problemas de ordem estratégica. E em vista do contexto abordado a pesquisa pode concluir que, é possível a aplicação da ferramenta BSC no curso de Mestrado Acadêmico em Administração da UECE, podendo ser adaptada às características específicas do curso e resultar num processo de melhoria.

Back, Dutra e Casagrande (2012) tendo seu trabalho denominado "Estruturação do balanced scorecard - bsc, para uma empresa do segmento varejista, com foco na avaliação do desempenho da ação estratégica de marketing promocional" com o objetivo de estruturar o Balanced Scorecard - BSC, utilizando apenas as perspectivas financeiras e do cliente, com foco na avaliação do desempenho da ação estratégica de marketing promocional, utilizando-o como ferramenta de gestão, controle e avaliação do desempenho organizacional.

Dentre as quatro perspectivas inerentes a ferramenta BSC as que possibilitaram com que a empresa pudesse realizar a avaliação do desempenho de sua estratégia foram as perspectivas financeira e perspectiva do cliente. Primeiramente é importante compreender que a ação de marketing promocional, sendo representada pela distribuição de panfletos promocionais, não é um objetivo estratégico, mas sim uma forma de atingir o objetivo de atrair e conquistar novos clientes, por exemplo. Para realizar o controle desta ação estratégica é importante dentro da atual estrutura do BSC da empresa verificar sua relação com as duas perspectivas em estudo.

Dentro da perspectiva financeira o principal objetivo foi aumentar a participação de mercado; sendo como indicador o percentual de participação do mercado; a situação atual share $3 \%$; e a meta consiste em alcançar $4 \%$ de participação de mercado; como iniciativa desenvolver ações estratégicas de marketing que permitem conquistar novos clientes. Dentro da perspectiva do cliente tem-se como objetivo 
expandir propaganda boca a boca; como indicadores o percentual de negócios oriundos da indicação de outros clientes e número de clientes que conhecem a marca da empresa; situação atual $15 \%$ e $0 \%$ (pesquisa não realizada); metas aumentar em $10 \%$ o número de negociações realizadas com novos clientes indicados por outros e desenvolver pesquisa de lembrança de marca; e as iniciativas são incentivar os atuais clientes a indicarem outros clientes através de descontos nas suas compras e desenvolver o roteiro.

Ao observar o painel de controle do BSC geral da organização verifica-se que a ação estratégica de panfletagem promocional, está em perfeita sintonia com os objetivos estratégicos estabelecidos, dentro da perspectiva financeira, uma vez que a ação aparece como iniciativa em dois dentre os cincos objetivos financeiros da empresa. Vale ressaltar ainda que o objetivo está sendo alcançado, dado que o percentual de participação de mercado cresceu $20 \%$ entre o período de maio a agosto. Um outro indicador importante e que tem relação direta com a ação em estudo é a receita oriunda de novos clientes, como os números revelam o faturamento da empresa cresceu $24 \%$, entende-se que este crescimento é proveniente de novos clientes que até a aplicação da ação não compravam na loja.

Na perspectiva do cliente, mesmo que a ação não esteja especificada nas iniciativas estratégicas, sabe-se que ela exerce influência e colabora muito na obtenção dos resultados desejados. A ação de panfletos promocionais está indiretamente ligada ao objetivo de expandir a propaganda boca a boca, cujo o indicador é o número de clientes que conhecem a marca. Ao receber panfletos em sua casa, nota-se que diversos consumidores chegavam na loja e afirmavam: "eu não conhecia a loja de vocês, nem sabia que existia." A distribuição dos panfletos promocionais pela região a qual a loja está localizada é uma operação fundamental para a percepção de valor do cliente quanto ao aspecto da imagem. Para medir a ação de panfletagem deve-se utilizar outra ação estratégica, uma pesquisa sobre a lembrança da marca na mente do consumidor e o número de clientes que conhecem a marca.

Percebe-se que a ação de panfletos promocionais gerou um bom resultado, registrando um crescimento de $24 \%$ nas vendas do 10 para o 2ㅇ quadrimestre. Destaca-se 0 aumento no percentual de vendas à vista com o mastercard, registrando um crescimento de $57 \%$. $A$ venda à vista como um todo teve um bom crescimento chegando a quase $22 \%$. Já a venda a prazo cresceu $28 \%$, o que é uma média interessante, uma vez que a venda a prazo representa receita futura.

Por fim Back, Dutra e Casagrande (2012) concluíram que o objetivo proposto pelo trabalho foi superado, pois além de elaborar uma proposta de avaliação do desempenho para a ação estratégica dos panfletos promocionais, considerando que a empresa não tinha nenhuma abordagem que enfrenta-se este problema, a pesquisa abordou o impacto que a ação gerou no faturamento, demostrando na prática, a influência da ação junto a perspectiva financeira.

Ferger e Alves (2015) através do estudo realizado e denominado como "Administração estratégica em agências de viagens e turismo: uma aplicação do Balanced Scorecard (BSC)" o qual o objetivo central da investigação foi descrever o processo e o resultado alcançado pela referida agência ao implementar o seu planejamento estratégico por meio do BSC. Como primeira etapa, os pesquisadores realizaram um levantamento de dados e informações, contemplando todos os departamentos da empresa, além disso para compreender o cenário com o máximo de profundidade foram realizadas entrevistas com a alta-direção (diretoria), gerentes dos departamentos focando em questões pertinentes à gestão, necessidades, panorama do clima organizacional e os resultados atuais esperados. Terminando o levantamento, os dados, resultados da auditoria e entrevistas, foram analisados com base nos indicadores financeiros e de mercado e tabulados e alocados pelos pesquisadores em cada uma das perspectivas do BSC.

Ao se analisar os dados verificou-se que um objetivo a ser perseguido tinha influencia ou era influenciado por outros. A fim de explicar a situação, um aspecto a ser melhorado correspondeu a desmotivação dos funcionários da empresa, a qual advinha da falta de estratégias de marketing, e também da falta de capacitação dos colaboradores e que impactava na falta de relacionamento com o cliente e na inexistência de procedimentos para tratar as reclamações, o que por fim, resultava na baixa rentabilidade nos resultados financeiros da empresa. Desta forma, ficou estabelecido em comum acordo que o objetivo a ser tratado (objetivo de curto prazo), após esta etapa seria o 
aumento da rentabilidade em até $7 \%$ em relação ao valor vigente.

Para cada uma das perspectivas do BSC foram elaborados objetivos específicos, os quais foram justificados pelos envolvidos na análise. $\mathrm{Na}$ perspectiva aprendizado a ação e objetivo foi o aumento do número de treinamento para os colaboradores, isso porque, seria fundamental contar com profissionais capacitados pois a qualificação do empregado gera consequentemente melhoria de produtividade e um grande diferencial para o negócio; na perspectiva de processos a ação e objetivo foi a redução do número de não conformidades de procedimentos operacionais; a perspectiva dos clientes a ação e objetivo foi a redução do índice de reclamações dos clientes; a perspectiva financeira teve como ação e objetivo a redução dos custos.

Após uma reunião, para cada perspectiva, ficaram definidos os indicadores e o prazo para sua mensuração que ficou determinado para ocorrer mensalmente. Os indicadores estabelecidos para a perspectiva de aprendizado foram a carga horária em treinamento e avaliação de eficácia em treinamento (ISO 10015); na perspectiva de processos determinou como indicador o percentual de não conformidades pelo determinado período, calculado pela quantidade de bilhetes de viagens que foram reemitidos e nas alterações realizadas nos pacotes de viagens já fechados; o indicador elencado para a perspectiva do cliente foi a porcentagem de reclamações considerando a quantidade de clientes atendidos; na perspectiva financeira foram previstas 3 escalas de redução, sendo que cada escala está associada a um mês com uma estimativa de $10 \%$ de redução. Após o terceiro mês, seria um total de $30 \%$ de redução. No quarto mês, o indicador deveria estabilizar em $30 \%$ de redução e, mantido nos meses subsequentes.

Figura 1. Diferenças e limitações entre os estudos de casos apresentados.

\begin{tabular}{|c|c|c|c|c|}
\hline \multicolumn{2}{|c|}{ Estudo de caso } & diferenças & limitações & semelhanças \\
\hline 1 & $\begin{array}{c}\text { OBalanced Scorecard aplicado no mestrado } \\
\text { acadêmico de Administração da Universidade } \\
\text { Estadual do Ceará - UECE. }\end{array}$ & $\begin{array}{c}\text { BSC no segmento Educacional, BSC } \\
\text { adapatado }\end{array}$ & $\begin{array}{c}\text { Aplicatdo apenas nos cursos de Pós - } \\
\text { graduação }\end{array}$ & resultados positivos \\
\hline 2 & $\begin{array}{c}\text { "Estruturação do balanced scorecard - bsc, para } \\
\text { uma empresa do segmento varejista, com foco } \\
\text { na avaliação do desempenho da ação } \\
\text { estratégica de marketing promocional" }\end{array}$ & $\begin{array}{c}\text { BSC no segmento Varejista, Foco em } \\
\text { atingir o cliente. }\end{array}$ & Não utilizou-se de todas perspectivas \\
\hline 3 & $\begin{array}{c}\text { "Administração estratégica em agências de } \\
\text { viagense turismo: uma aplicação do Balanced } \\
\text { Scorecard (BSC)" }\end{array}$ & $\begin{array}{c}\text { BSC no segmento de Turismo, } \\
\text { treinamento na ISO10015 }\end{array}$ & Necessitou de ferramentas auxiliares, \\
falta de capacitação & \\
\hline
\end{tabular}

Fonte: (Autoria Própria, 2018).

Os resultados alcançados com 0 planejamento estratégico na agência de turismo dentro da perspectiva aprendizado obteve $92 \%$ de carga horária de treinamento e $96 \%$ de eficácia atingida. Na perspectiva processos gerou $0,3 \%$ de não conformidades. Por conseguinte, a perspectiva dos clientes atingiu $0,9 \%$ de reclamações de clientes considerando o número de clientes atendidos. Por fim a perspectiva financeira resultou em $29,4 \%$ de redução. Verificou-se que com a aplicação da ferramenta $B S C$, obteve-se aumento da produtividade e a melhoria da qualidade dos serviços, contribuindo de maneira geral para o aumento da rentabilidade em $5 \%$.
Quanto ao alcance dos objetivos considera-se que o central foi atendido, visto que foi possível identificar os procedimentos adotados pela empresa para a aplicação da ferramenta BSC para o gerenciamento da estratégia. Verificou-se que a ferramenta seria útil no sentido de direcionar as ações da empresa, no entanto, para a análise foram necessários instrumentos auxiliares específicos como diagrama de causa e efeito, além disso, na implementação do PDCA que foi de grande valia como instrumento auxiliar.

Assim como é representado pela Figura 1, com relação as semelhanças todos os estudos de caso apresentados obtiveram resultados positivos, com uma ressalva no segundo caso, 
onde os resultados obtidos superaram as expectativas. Assim observa-se que o método BSC foi eficaz em todos esses casos, mostrando sua capacidade de direcionar a estratégia de qualquer empresa, uma vez que todas as empresas estudadas são de diferentes segmentos. No que se diz respeito às diferenças, no primeiro caso houve uma adaptação da perspectiva financeira por outra que melhor se adequasse ao setor educacional sendo a perspectiva de desempenho acadêmico, no segundo caso utilizaram-se apenas duas perspectivas, e no terceiro caso houve uma necessidade de treinamento na ISO 10015. Isto demonstra a flexibilidade do BSC, podendo assim, as organizações adaptar a sua estrutura de acordo com o ambiente a qual está inserida, mesmo que não seja aplicada integralmente (utilizando todas as perspectivas), se faz viável este tipo de modificação, uma vez que permitiram a geração de resultados satisfatórios. Por fim cada estudo demonstrou sua limitação, sendo que no primeiro caso o BSC teve como escopo apenas os cursos de pós-graduação, já no segundo caso utilizou-se apenas perspectivas do cliente e financeiras e no terceiro caso foi necessário utilização de ferramentas auxiliares, como PDCA e Diagrama de Ishikawa.

\section{CONSIDERAÇÕES FINAIS}

Neste trabalho primeiramente abordouse uma revisão bibliográfica a respeito do BSC buscando trazer seus conceitos e os aspectos históricos desta ferramenta para que deste modo o leitor possa adquirir conhecimento, para isto o estudo trouxe diferentes formas de abordagem por meio da apresentação de três estudos de casos em segmentos diferentes, cada um com sua peculiaridade e suas respectivas adaptações e resultados. Por fim realizou-se uma análise dos aspectos semelhantes, distintos e limitações dos estudos, buscando assim avaliar como a ferramenta BSC foi utilizada.

A limitação do presente trabalho se dá pelo fato de ter utilizado apenas três estudos de caso. E para estudos futuros sugere-se a abordagem de um número maior de estudos de caso em segmentos diversos e/ou segmentos específicos.

\section{REFERÊNCIAS}

BACK, L.; DUTRA, A.; CASAGRANDE, J. L. Estruturação do balanced scorecard (BSC) para uma empresa do segmento varejista, com foco na avaliação do desempenho da ação estratégica de marketing promocional. ConTexto, v. 12, n. 22, p. 7-25, jul./dez. 2012.

BHALLA, Ajay et al. Exploring alternative strategic management paradigms in high-growth ethnic and non-ethnic family firms. Small Business Economics, v. 32, n. 1, p. 77-94, 2009.

BIASOTTO, Eduardo et al. Aplicação do BSC na gestão da TPM: estudo de caso em indústria de processo. Dissertação (mestrado) - Universidade Federal de Santa Catarina, Centro Tecnológico. Programa de Pós-Graduação em Engenharia Mecânica, Florianópolis, 2006.

BORGES, R. C. O.; ALMEIDA, F. A. M.; SILVEIRA, M. L. $O$ balanced scorecard aplicado no mestrado acadêmico de administração da universidade estadual do ceará - UECE. Monografia (Graduação) - Acadêmia de Curso de Bacharelado em Administração da Faculdade Cearense. Fortaleza, 2011.

CARVALHO, M. M.; LAURINDO, F. J. B. Estratégia competitiva: dos conceitos a implementação. 2 . ed. São Paulo: Atlas, 2010.

DAVIS, Stan; ALBRIGHT, Tom. An investigation of the effect of balanced scorecard implementation on financial performance. Management accounting research, v. 15, n. 2 , p. 135-153, 2004. https://doi.org/10.1016/i.mar.2003.11.001

DE FARIA, A. C.; DA COSTA, M. F. G. Gestão de custos logísticos: custeio baseado em atividades $(A B C)$, balanced scorecard (BSC), valor econômico agregado (EVA). Atlas, 2007.

FEGER, J. E.; ALVES, P. A. Administração estratégica em agência de viagens e turismo: uma aplicação do Balanced Scorecard (BSC). Turismo e Sociedade, v. 8, n. 3, 2016.

KAPLAN, Robert S.; NORTON, David P.A estratégia em ação: balanced scorecard. Gulf Professional Publishing, 1997. 
KAPLAN, R. S.; NORTON, D. P. Organização orientada para a estratégia: como as empresas que adotam o balanced scorecard prosperam no novo ambiente de negócios. Gulf Professional Publishing, 2000.

LIMA, Sílvia Aparecida Pereira. Sobre a Importância da Estratégia Corporativa Para as Organizações. Revista Científica Eletrônica de Administração, p. 1-4. 2009.

MAINARDES, E. W.; FERREIRA, J.; raposo, m. Conceitos de estratégia e gestão estratégica: qual é o nível de conhecimento adquirido pelos estudantes de gestão?. FACEF PESQUISA. Franca, v.14, n. 3, p. 278-298, 2011.

OLIVEIRA, M. F. Balanced scorecard: uma análise da produção acadêmica brasileira na área de administração. Tese de Doutorado. Dissertação (Mestrado em Administração). Universidade Federal de Lavras. Minas Gerais, 2008.

SIBBET, David. 75 years of management ideas and practice 1922-1997. Harvard Business Review, v. 75, n. 5, p. 2-12, 1997.

SPECKBACHER, Gerhard; BISCHOF, Juergen; PFEIFFER, Thomas. A descriptive analysis on the implementation of balanced scorecards in German-speaking countries. Management accounting research, v. 14 , n. 4, p. 361-388, 2003. https://doi.org/10.1016/i.mar.2003.10.001 